\title{
Zur „Managementforschung“
}

Ziel der „Managementforschung“" ist es, einen Überblick über den aktuellen Stand und Ergebnisse der Forschung zu Managementproblemen zu geben; zugleich soll sie ein Diskussionsforum für neue Trends und Strömungen sein. Die „Managementforschung“ richtet sich an Forscher und Studierende der Wirtschafts- und Sozialwissenschaften sowie an wissenschaftlich interessierte Praktiker und Managementtrainer.

Die „Managementforschung“ ist am Institut für Management der Freien Universität Berlin entstanden und erscheint seit 1991 jährlich. Sie wird von Georg Schreyögg, Peter Conrad und Jörg Sydow herausgegeben (GründungsMitherausgeber Wolfgang H. Staehle $\dagger$ ). Der Schwerpunkt liegt auf innovativen Forschungsbeiträgen zu zentralen Gebieten der Managementforschung. Neben anerkannten Fachvertretern haben auch qualifizierte Nachwuchswissenschaftler die Gelegenheit, zu aktuellen Fragen Stellung zu nehmen. Die „Managementforschung“ versteht sich als ein Publikationsorgan für Wissenschaftler, die Management als Funktion und Institution untersuchen. Disziplinäre Offenheit ist Programm; sie wird durch die personelle Besetzung des Beirats unterstrichen, der die Herausgeber bei der Akquisition, Begutachtung und Auswahl geeigneter Beiträge unterstützt. Dem Herausgeberbeirat gehören zur Zeit an:

aus dem Bereich Betriebswirtschaftslehre:

- Prof. Dr. Joachim Griese, Universität Bern

- Prof. Dr. Oskar Grün, Wirtschaftsuniversität Wien

- Prof. Dr. Werner R. Müller, Universität Basel

- Prof. Dr. Gilbert Probst, Universität Genf

- Prof. Dr. Dieter Sadowski, Universität Trier

- Prof. Dr. Bernd Schauenberg, Universität Freiburg

- Prof. Dr. Martin K. Welge, Universität Dortmund

aus dem Bereich der Arbeits- und Organisationspsychologie

- Prof. Dr. Oswald Neuberger, Universität Augsburg

aus dem Bereich der Arbeits- und Organisationssoziologie und Politologie

- Prof. Dr. Walther Müller-Jentsch, Ruhr-Universität Bochum

- Prof. Dr. Frieder Naschold, Freie Universität und WZB Berlin

- Prof. Dr. Helmut Willke, Universität Bielefeld

Beiträge werden von Autoren entweder unaufgefordert bei den Herausgebern zur Publikation eingereicht oder bei den Autoren gezielt eingeworben. Grundsätzlich wird die erste Manuskriptfassung eines jeden Beitrags einem anonymen „doppelt blinden“ Reviewprozeß unterzogen. Regelmäßig sind an der Begutachtung eines Beitrags Vertreter unterschiedlicher Disziplinen beteiligt. 
VI Zur „Managementforschung”

Auf der Grundlage ausfuhrlicher Reviews wird uber die Akzeptanz sowie über Art und Umfang der gewünschten Überarbeitung des Manuskriptes entschieden. Jeder Band der „Managementforschung“ ist somit das Ergebnis einer engen Kooperation zwischen Autoren, Beiträgen und Herausgebern. 